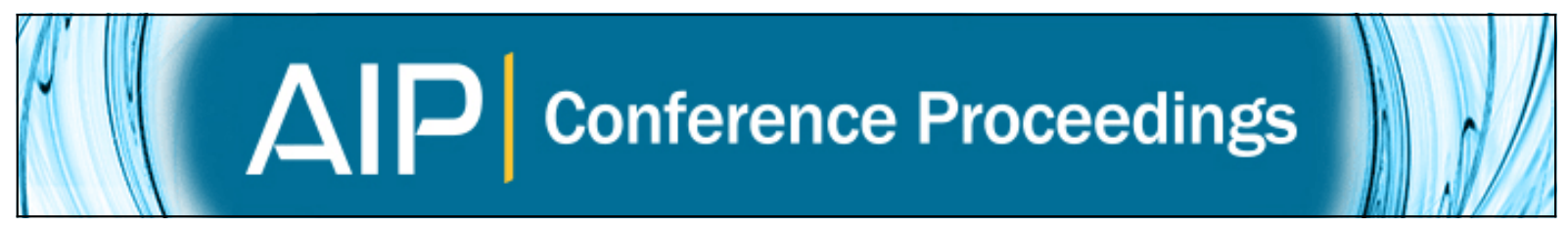

\title{
A finite difference, multipoint flux numerical approach to flow in porous media: Numerical examples
}

Hossam Osman, Amgad Salama, Shuyu Sun, and Kai Bao

Citation: AIP Conference Proceedings 1453, 217 (2012); doi: 10.1063/1.4711178

View online: http://dx.doi.org/10.1063/1.4711178

View Table of Contents: http://scitation.aip.org/content/aip/proceeding/aipcp/1453?ver=pdfcov

Published by the AIP Publishing

\section{Articles you may be interested in}

Rarefied gas flow through porous media: Experiments and numerical simulation

AIP Conf. Proc. 1501, 1202 (2012); 10.1063/1.4769678

Numerical investigation of flow through porous media using lattice Boltzmann method

AIP Conf. Proc. 1440, 863 (2012); 10.1063/1.4704297

Influence of microbial biofilms on reactive transport in porous media

AIP Conf. Proc. 1453, 276 (2012); 10.1063/1.4711188

A finite volume method for the solution of fluid flows coupled with the mechanical behavior of compacting porous media

AIP Conf. Proc. 1453, 205 (2012); 10.1063/1.4711176

A novel numerical approach for the solution of the problem of two-phase, immiscible flow in porous media: Application to LNAPL and DNAPL

AIP Conf. Proc. 1453, 135 (2012); 10.1063/1.4711165 


\title{
A Finite Difference, Multipoint Flux Numerical Approach to Flow in Porous Media: Numerical Examples
}

\author{
Hossam Osman, Amgad Salama, Shuyu Sun and Kai Bao \\ Computational Transport Phenomena Laboratory, Division of Mathematics and Computer Science and \\ Engineering, King Abdullah University of Science and Technology
}

\begin{abstract}
It is clear that none of the current available numerical schemes which may be adopted to solve transport phenomena in porous media fulfill all the required robustness conditions. That is while the finite difference methods are the simplest of all, they face several difficulties in complex geometries and anisotropic media. On the other hand, while finite element methods are well suited to complex geometries and can deal with anisotropic media, they are more involved in coding and usually require more execution time. Therefore, in this work we try to combine some features of the finite element technique, namely its ability to work with anisotropic media with the finite difference approach. We reduce the multipoint flux, mixed finite element technique through some quadrature rules to an equivalent cell-centered finite difference approximation. We show examples on using this technique to single-phase flow in anisotropic porous media.
\end{abstract}

Keywords: Cell-centered Finite Difference, Finite Element, Multipoint Flux, Quadrature rule, Anisotropy PACS: $02.60 . \mathrm{Cb}, 02.70 . \mathrm{Bf}, 02.70 . \mathrm{Dh}$

\section{INTRODUCTION}

Petroleum reservoirs are generally recognised to exhibit heterogeneity (spatial variations in rock property) and anisotropy (unequal values of properties with direction at any given location). This is particularly true of Middleeastern carbonate reservoirs which are highly heterogeneous [1]. A crucial parameter for hydrocarbon production is the permeability, which in most reservoirs exhibit anisotropy, see Figure 1. In this case permeability is described as a full tensor rather than a simple scalar quantity. Permeability anisotropy causes the drainage area around wells to be elliptical [2], see Figure 2. Elongated drainage increases production intereference and drainage overlap between adjacent wells, potentially leaving parts of the reservoir undrained. It has also been shown that in reservoirs with permeability anisotropy, particularly in naturally fractured reservoirs, induced fractures grow parallel to the natural fractures [3]. From the standpoint of well performace however, fractures that grow perpendicular to the direction of natural fractures show the greatest positive impact in well performance. Knowledge of the orientation and magnitude of anisotropy is therefore an economically significant factor in the development and management of reservoirs. The geology of the reservoir which may include faults and non-parallel layers pose major challenges to existing models. To account for anisotropy, models may need to use non-orthogonal grids, with a full permeability tensor which may be discontinuous in the discretisation of the flow equations. Classical reservoir simulators which adopt the cell-centered finite difference five or seven point stencil (for two or three dimensions, respectively) can not handle full tensors.

The multipoint point flux approximation (MPFA) method has been developed by the oil industry to be the next generation method in modeling flow in porous media which accommodates the use of full tensors in the discretisation. The MPFA method also preserves the mathematical model's local mass conservation, i.e. the sum of the fluxes over each cell's edges equals to the accumulation term plus any sources/sinks and that the flux is continuous across each edge.

In this paper we aim to develop the MPFA method as a perturbed Mixed Finite Element method. This is then reduced to an equivalent cell-centered finite difference approximation by applying a quadrature rule. This work is motivated by work conducted by Aavatsmark in [4] and Wheeler and Yotov [5]. Various interesting examples were then investigated emphasising the method's capability to handle inhomogeneous, anisotropic media.

\section{MATHEMATICAL FORMULATION}

The governing equations describing flow and transport in porous media are based on the continuum approach in which field variables are continuous functions of space and time, Salama and van Geel [7], [8]. Consider the second order elliptic problem that models single phase flow in porous media. The problem can be written as a 


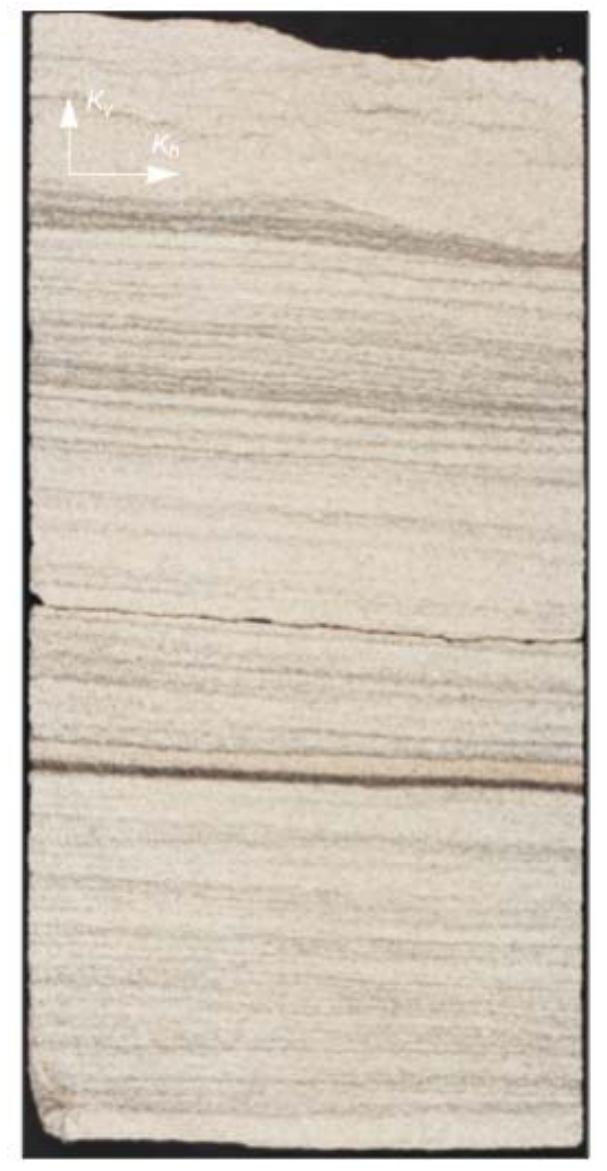

FIGURE 1. Permeability anisotropy in sandstone. Thin layers of shale and quartz retard flow in the vertical direction, making horizontal permeability much greater than the vertical permeability, from [6].

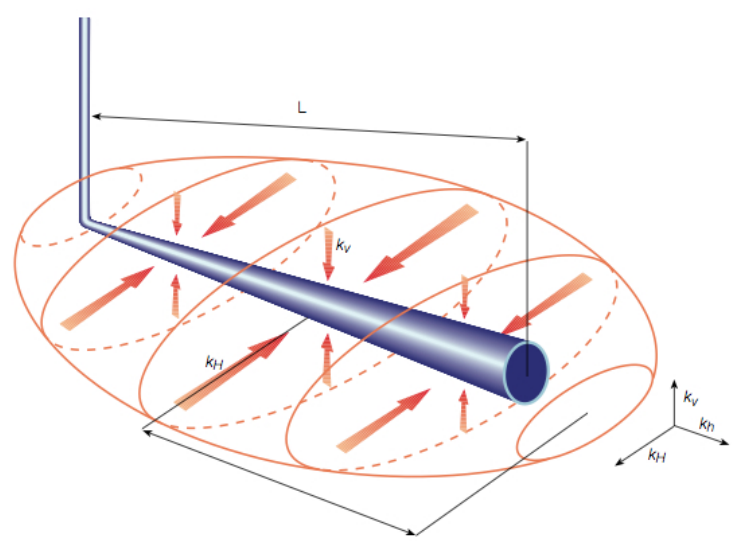

FIGURE 2. Anisotropy causes drainage area around wells to be elliptical, here the horizontal permeability is much greater than the vertical permeability, from [6]. system of two first order equations

$$
\begin{array}{cc}
\mathbf{u}=-K \nabla p & \text { in } \Omega, \\
\nabla \cdot \mathbf{u}=f & \text { in } \Omega, \\
p=p_{B} & \text { on } \delta D, \\
\mathbf{u} \cdot \mathbf{n}=0 & \text { on } \delta N,
\end{array}
$$

where the domain $\Omega \subset \mathbf{R}^{\mathbf{d}}, d=2$ or 3 , has a boundary $\delta \Omega=\delta D \cup \delta N, \delta D \cap \delta N=\mathbf{0}, \mathbf{n}$ is the outward unit normal on $\delta \Omega$ and $\mathrm{K}$ is a symmetric positive definite tensor.

For flow in porous media $p$ and $\mathbf{u}$ are the pressure and darcy velocity respectively and $\mathrm{K}$ represents the ratio of the media's permeability to the fluid's viscosity. Next the mixed variation form of the above system will be derived.

Let $\mathscr{L}$ denote the Lebesgue square-integrable functions on the domain $\Omega \subset \mathbf{R}^{\mathbf{d}}$ with inner product $(\cdot, \cdot)$. Also use the space:

$$
\mathbf{H}(\operatorname{div}, \Omega)=\left\{\mathbf{v} \in\left(\mathscr{L}^{2}(\Omega)\right)^{2}: \nabla \cdot \mathbf{v} \in \mathscr{L}^{2}(\Omega)\right\}
$$

Define:

$$
\mathbf{V}=\mathbf{H}(\operatorname{div}, \Omega), \quad W=\mathscr{L}^{2}(\Omega)
$$

Multiply Eq. 1 by $\mathbf{v} \in \mathbf{V}$ and integrate over $\Omega$ to get:

$$
\left(K^{-1} \mathbf{u}, \mathbf{v}\right)=(p, \nabla \cdot \mathbf{v})-\left\langle p_{B}, \mathbf{v} \cdot \mathbf{n}\right\rangle
$$

Where the Green's formula was used to expand the $(\mathbf{v}, \nabla p)$ term. Similarly by multiplying Eq. 2 by any $\omega \in W$ results in:

$$
(\nabla \cdot \mathbf{u}, \omega)=(f, \omega)
$$

The above second order elliptic system therefore becomes:

$$
\begin{array}{rr}
\left(K^{-1} \mathbf{u}, \mathbf{v}\right)=(p, \nabla \cdot \mathbf{v})-\left\langle p_{B}, \mathbf{v} \cdot \mathbf{n}\right\rangle_{\delta D}, & \mathbf{v} \in \mathbf{V}, \\
(\nabla \cdot \mathbf{u}, \omega)=(f, \omega), & \omega \in W,
\end{array}
$$

This is known as the mixed variation form or the weak form of Eqs. 1 - 4. If $\mathbf{u}$ and $p$ satisfy Eqs. 1 and 2 they will also satisfy Eqs. 9 and 10. The opposite holds as long as $p$ is sufficiently smooth.

Now let $\mathbf{V}$ be approximated by a finite dimensional space $\mathbf{V}_{h} \subset \mathbf{V}$ and $W$ by $W_{h} \subset W$. The MFEM approximation of Eqs. 9 and 10 is then:

Find $\mathbf{u}_{h} \in \mathbf{V}_{h}$ and $p_{h} \in W_{h}$ such that

$$
\begin{array}{rr}
\left(K^{-1} \mathbf{u}_{h}, \mathbf{v}\right)=\left(p_{h}, \nabla \cdot \mathbf{v}\right)-\left\langle p_{B}, \mathbf{v} \cdot \mathbf{n}\right\rangle_{\delta D}, & \mathbf{v} \in \mathbf{V}_{h}, \\
\left(\nabla \cdot \mathbf{u}_{h}, \omega\right)=(f, \omega), & \omega \in W_{h},
\end{array}
$$

\section{Rectangular element spaces}

The element spaces $\mathbf{V}_{h}$ and $W_{h}$ can not be chosen independently and for the problem Eqs. 11 and 12 to have 
a unique solution a discrete inf-sup condition, known as the Babuška-Brezzi condition must be imposed:

$$
\sup _{0 \neq \mathbf{v} \in \mathbf{v}} \frac{|(\nabla \cdot \mathbf{v}, \omega)|}{\|\mathbf{v}\| \mathbf{v}} \geq C_{2}\|\omega\| \quad \forall \in W_{h}
$$

where $C_{2}>0$ is independent of the maximum element edge length, $h$ and $\|\cdot\|$ denotes the $\mathscr{L}^{2}$ norm.

For this work the mixed finite element spaces $\mathbf{V}_{h}$ and $W_{h}$ were considered over rectangles. First let us consider the case where $\Omega$ is a rectangular domain. Let $\left\{\tau_{h}\right\}$ denote a family of partitions of $\Omega$ into rectangular subdomains or cells, where $h$ is the maximum element edge length such that the horizontal and vertical edges are parallel to the $\mathrm{x}$ - and $\mathrm{y}$ - coordinate axes respectively and that adjacent elements share a common edge. Now define:

$$
\begin{array}{r}
Q_{l, r}(E)=\left\{v: v(\mathbf{x})=\sum_{i=o}^{l} \sum_{j=0}^{r} v_{i j} x^{i} y^{j},\right. \\
\left.\mathbf{x}=(x, y) \in E, v_{i j} \in R\right\}
\end{array}
$$

i.e. $Q_{l, r}(E)$ is the space of polynomials of degree at most $l$ in $x$ and $r$ in $y$ where $l, r \geq 0$.

The BDM spaces on rectangles differ considerably to the RT spaces on rectangles; the vector elements are based on augmenting the space of vector polynomials of total degree $r$ by an additional two vectors in place of augmenting the space of vector tensor-products of polynomials of degree $r$ by $2 r+2$ polynomials of higher degree. A lower dimensional space for the scalar variable is also used [9]. These spaces, for any $r \geq 1$

$$
\begin{array}{r}
\mathbf{V}_{h}(E)=\left(P_{r}(E)\right)^{2} \oplus \operatorname{span}\left\{\operatorname{curl}\left(x^{r+1} y\right), \operatorname{curl}\left(x y^{r+1}\right)\right\} \\
W_{h}=P_{r-1}(E)
\end{array}
$$

The lowest order $B D M_{1}$ mixed finite element spaces are defined as

$$
\begin{array}{r}
\mathbf{V}_{h}=\left\{v: v=\left(a_{E}^{1}+a_{E}^{2} x+a_{E}^{3} y-a_{E}^{4} x^{2}-2 a_{E}^{5} x y, \ldots\right.\right. \\
\left.a_{E}^{6}+a_{E}^{7} x+a_{E}^{8} y+2 a_{E}^{4} x y+a_{E}^{5} y^{2}\right), \\
\left.a_{E}^{i} \in R, i=1,2, \ldots, 8\right\}, \\
W_{h}=P_{0}(E),
\end{array}
$$

Its dimension is eight. The degrees of freedom for $\mathbf{V}_{h}$ are the values of the normal components at any two points on each edge of the element. We chose to place these points at the vertices of each edge.

\section{Two point flux approximation method, TPFA}

For comparison the two point flux approximation cellcentered finite difference method was developed along side the multipoint flux approximation method with quadrature rule.

In order to highlight the features of this scheme, a simple $2 \mathrm{D}$ flow problem in a porous medium over a rectangular domain was considered. The governing equations again are:

$$
\begin{array}{cc}
\mathbf{u}=-K \nabla p & \text { in } \Omega, \\
\nabla \cdot \mathbf{u}=f & \text { in } \Omega, \\
p=p_{B} & \text { on } \delta D, \\
\mathbf{u} \cdot \mathbf{n}=0 & \text { on } \delta N,
\end{array}
$$

Using the cell-centered approach the above system is discretised over a 2D mesh (Figure 3 ) to give the following for Eq. 19:

$$
\begin{array}{r}
u_{x}^{\left(i, j+\frac{1}{2}\right)}=-K_{x x}^{\left(i, j+\frac{1}{2}\right)} \frac{\Delta_{x} P^{\left(i+\frac{1}{2}, j+\frac{1}{2}\right)}}{\Delta x^{\left(i+\frac{1}{2}\right)}} \\
u_{x}^{\left(i+1, j+\frac{1}{2}\right)}=-K_{x x}^{\left(i+1, j+\frac{1}{2}\right)} \frac{\Delta_{x} P^{\left(i+\frac{3}{2}, j+\frac{1}{2}\right)}}{\Delta x^{\left(i+\frac{3}{2}\right)}}
\end{array}
$$

where $i=1 \ldots m+1, j=1 \ldots n, \Delta_{x} P^{(i, j)}=P^{(i, j)}-P^{(i-1, j)}$ and $\Delta x^{(i)}=x^{(i)}-x^{(i-1)}$

$$
\begin{array}{r}
u_{y}^{\left(i+\frac{1}{2}, j\right)}=-K_{y y}^{\left(i+\frac{1}{2}, j\right)} \frac{\Delta_{y} P^{\left(i+\frac{1}{2}, j+\frac{1}{2}\right)}}{\Delta y^{\left(j+\frac{1}{2}\right)}} \\
u_{y}^{\left(i+\frac{1}{2}, j+1\right)}=-K_{y y}^{\left(i+\frac{1}{2}, j+1\right)} \frac{\Delta_{y} P^{\left(i+\frac{1}{2}, j+\frac{3}{2}\right)}}{\Delta y^{\left(j+\frac{3}{2}\right)}}
\end{array}
$$

where $i=1 \ldots m, j=1 \ldots n+1, \Delta_{y} P^{(i, j)}=P^{(i, j)}-P^{(i, j-1)}$ and $\Delta y^{(j)}=y^{(j)}-y^{(j-1)}$

The dicretisation of Eq. 20 yields:

$$
\frac{u_{x}^{\left(i+1, j+\frac{1}{2}\right)}-u_{x}^{\left(i, j+\frac{1}{2}\right)}}{\Delta x^{(i+1)}}+\frac{u_{y}^{\left(i+\frac{1}{2}, j+1\right)}-u_{y}^{\left(i+\frac{1}{2}, j\right)}}{\Delta y^{(j+1)}}=q^{\left(i+\frac{1}{2}, j+\frac{1}{2}\right)}
$$

where $i=1 \ldots m, j=1 \ldots n$

Now by substituting Eqs. 23 - 26 into Eq. 27, the velocity unknowns can be eliminated resulting in an equation in pressure only which may be solved numerically to obtain the pressure field (Eq. 28). Using back substitution the velocity field can then be found.

$$
\begin{array}{r}
\frac{K_{x x}^{\left(i, j+\frac{1}{2}\right)} \frac{\Delta_{x} P^{\left(i+\frac{1}{2}, j+\frac{1}{2}\right)}}{\Delta x^{\left(i+\frac{1}{2}\right)}}-K_{x x}^{\left(i+1, j+\frac{1}{2}\right)} \frac{\Delta_{x} P^{\left(i+\frac{3}{2}, j+\frac{1}{2}\right)}}{\Delta x^{\left(i+\frac{3}{2}\right)}}}{\Delta x^{(i+1)}} \\
-\frac{K_{y y}^{\left(i+\frac{1}{2}, j\right)} \frac{\Delta_{y} P^{\left(i+\frac{1}{2}, j+\frac{1}{2}\right)}}{\Delta y^{\left(j+\frac{1}{2}\right)}}-K_{y y}^{\left(i+\frac{1}{2}, j+1\right)} \frac{\Delta_{y} P^{\left(i+\frac{1}{2}, j+\frac{3}{2}\right)}}{y^{\left(j+\frac{3}{2}\right)}-x^{\left(j+\frac{1}{2}\right)}}}{\Delta y^{(j+1)}}=q^{\left(i+\frac{1}{2}, j+\frac{1}{2}\right)}
\end{array}
$$




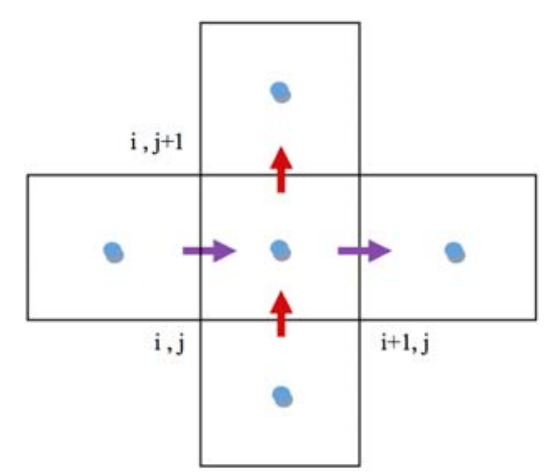

FIGURE 3. 2D non uniform cell-centered mesh

where $i=1 \ldots m, j=1 \ldots n$

Notice that for each velocity component in each cell two pressure values are needed hence the name 'two point flux'. Also notice the final form of this method in Eq. 28 utilises five pressures, this creates what was described earlier as the 'five point stencil' where the divergence across each cell is dependent upon the pressure in that cell and the four surrounding cells as shown in Figure 3.

\section{The Multipoint flux approximation, MPFA method}

The MPFA approach is based on a control-volume formulation of the Darcy equation where more than two pressure values are used in the approximation of the flux for each edge of the control-volume or cell. The basic idea here is to divide each grid cell into subcells and assume linear variation of the pressure in each subcell. All subcells with a common corner form an interaction volume as shown in Figure 4. For the case of a rectangular (or quadrilateral) mesh the interaction volume will contain four subcells and hence four subedges.

\section{The quadrature rule}

To obtain the MPFA method as a MFE method quadrature rules will be defined. $\mathbf{K}_{E}$ will be evaluated at the midpoint of the cell and the components of $\mathbf{K}_{E}^{-1}$ are denoted by $\kappa_{i j}^{E}, j, k=1,2$.

For $\mathbf{q}, \mathbf{v} \in \mathbf{V}_{h}$, define the quadrature rule on a quadrilateral element:

$\left(K^{-1} \mathbf{q}, \mathbf{v}\right)_{Q}=\sum_{E \in \mathscr{T}_{h}}\left(K^{-1} \mathbf{q}, \mathbf{v}\right)_{Q, E}=\frac{1}{2} \sum_{i=1}^{4} \sum_{j, k=1}^{2}\left|E_{i}\right| \kappa_{i j}^{E_{i}} q_{i j} v_{i j}$

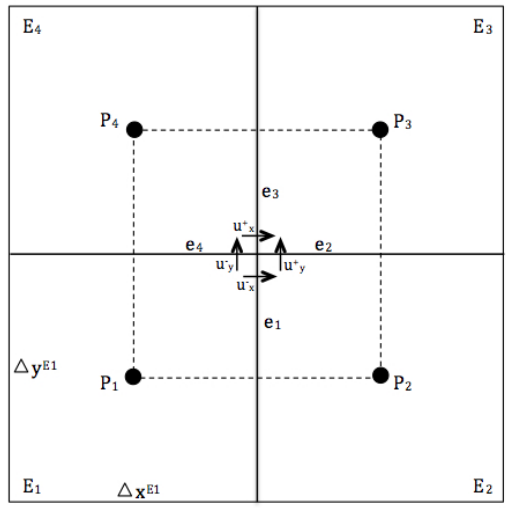

FIGURE 4. Four elements (solid line) sharing a vertex, creating an interaction volume (dotted line). The dots denote the cell pressures and arrows denote the normal velocities on the subcell edges.

The corner vector $\mathbf{q}\left(\mathbf{r}_{i}\right)$ is uniquely determined by it's normal components to the two edges sharing a vertex. Let us denote the basis functions associated with $\mathbf{r}_{i}$ by $\mathbf{v}_{i j}, j=1,2$ imposing the following conditions:

$$
\begin{array}{r}
\left(\mathbf{v}_{i j} \cdot \mathbf{n}_{i j}\right)\left(\mathbf{r}_{i}\right)=1, \quad\left(\mathbf{v}_{i j} \cdot \mathbf{n}_{i k}\right)\left(\mathbf{r}_{i}\right)=0, k \neq j, \\
\left(\mathbf{v}_{i j} \cdot \mathbf{n}_{l k}\right)\left(\mathbf{r}_{l}\right)=0, l \neq i, k=1,2
\end{array}
$$

Clearly the quadrature rule Eq. 29 only couples the two basis functions associated with a corner, e.g.

$$
\begin{aligned}
& \left(\mathbf{K}^{-1} \mathbf{v}_{11}, \mathbf{v}_{11}\right)_{Q, E_{1}}=\frac{\kappa_{11}^{E_{1}} \Delta x^{E_{1}} \Delta y^{E_{1}}}{2} \\
& \left(\mathbf{K}^{-1} \mathbf{v}_{11}, \mathbf{v}_{12}\right)_{Q, E_{1}}=\frac{\kappa_{12}^{E_{1}} \Delta x^{E_{2}} \Delta y^{E_{2}}}{2} \\
& \left(\mathbf{K}^{-1} \mathbf{v}_{11}, \mathbf{v}_{i j}\right)_{Q, E_{1}}=0, \forall i j \neq 11,12
\end{aligned}
$$

\section{Cell-centered finite difference approximation}

The Multipoint Flux Mixed Finite Element method reduces to a cell-centered system for the pressures. Consider an internal vertex $\mathrm{r}$ which is shared by elements $E_{i}, i=1, \ldots, 4$, see Figure 4 and denoting the edges that share the vertex by $e_{i}, i=1, \ldots, 4$, the velocity basis functions on these edges associated with the vertex by $\mathbf{v}_{i}$, $i=1, \ldots, 4$ and the corresponding values of the normal components of $\mathbf{u}_{\mathbf{h}}$ by $u_{x}^{-}, u_{y}^{+}, u_{x}^{+}, u_{y}^{-}$.

Taking for example $\mathbf{v}=\mathbf{v}_{1}$ in the quadrature rule; due to the locality of the basis functions interaction, $u_{x}^{-}$ will only couple with $u_{y}^{+}$and $u_{y}^{-}$. This leads to a $4 \times 4$ linear system for $u_{x}^{-}, \ldots, u_{y}^{-}$by taking $\mathbf{v}=\mathbf{v}_{1}, \ldots, \mathbf{v}_{4}$. The coefficients of the linear system are given by:

$$
a_{i j}=\left(\mathbf{K}^{-1} \mathbf{v}_{i}, \mathbf{v}_{j}\right)_{Q}, i, j=1, \ldots, 4
$$


Inverting this $4 \times 4$ linear system allows us to express the velocities in terms of the cell-centered pressures. Substituting these expressions into the mass conservation equation forms the cell-centered stencil. The pressure in element $\mathrm{E}$ is coupled with the pressures in the elements that share a vertex with E; on a rectangular grid this leads to a 9-point stencil.

As an example lets take $\mathbf{v}=\mathbf{v}_{1}$ in Eq. 12 for any internal vertex. The first term we get:

$$
\begin{aligned}
\left(\mathbf{K}^{-1} \mathbf{u}_{h}, \mathbf{v}_{1}\right)_{Q}= & \left(\mathbf{K}^{-1} \mathbf{u}_{h}, \mathbf{v}_{1}\right)_{Q, E_{1}}+\left(\mathbf{K}^{-1} \mathbf{u}_{h}, \mathbf{v}_{1}\right)_{Q, E_{2}} \\
= & \frac{1}{2}\left(\kappa_{11}^{E_{1}} u_{x}^{-} A^{E_{1}}+\kappa_{12}^{E_{1}} u_{y}^{-} A^{E_{2}}\right) \\
& +\frac{1}{2}\left(\kappa_{11}^{E_{2}} u_{x}^{-} A^{E_{2}}+\kappa_{12}^{E_{2}} u_{y}^{+} A^{E_{1}}\right)
\end{aligned}
$$

where $A^{E_{i}}=\Delta x^{E_{i}} \Delta y^{E_{i}}$

The second term can be written as:

$$
\begin{aligned}
\left(p_{h}, \nabla \cdot \mathbf{v}_{1}\right) & =\left(p_{h}, \nabla \cdot \mathbf{v}_{1}\right)_{E_{1}}+\left(p_{h}, \nabla \cdot \mathbf{v}_{1}\right)_{E_{2}} \\
& =\left\langle p_{h}, \mathbf{v}_{1} \cdot \mathbf{n}_{E_{1}}\right\rangle_{e_{1}}+\left\langle p_{h}, \mathbf{v}_{1} \cdot \mathbf{n}_{E_{2}}\right\rangle_{e_{2}} \\
& =\frac{1}{2}\left(p_{1}-p_{2}\right) \Delta y^{E_{1}}
\end{aligned}
$$

The third term is obviously zero for internal vertices. By constructing similar equations for the other three basis functions the linear system can then be written in the following algebraic form:

$$
\mathbf{A}\left[u_{x}^{-}, u_{y}^{+}, u_{x}^{+}, u_{y}^{-}\right]^{T}=\mathbf{B}\left[p_{1}, p_{2}, p_{3}, p_{4}\right]^{T}
$$

where

$$
\begin{gathered}
\mathbf{A}=\frac{1}{2}\left[\begin{array}{cccc}
\kappa_{11}^{E_{1}}\left(A^{E_{1}}+A^{E_{2}}\right) & \kappa_{12}^{E_{2}} A^{E_{2}} & 0 & \kappa_{12}^{E_{1}} A^{E_{1}} \\
\kappa_{12}^{E_{2}} A^{E_{2}} & \kappa_{22}^{E_{2}}\left(A^{E_{2}}+A^{E_{3}}\right) & \kappa_{12}^{E_{3}} A^{E_{3}} & 0 \\
0 & \kappa_{12}^{E_{3}} A^{E_{3}} & \kappa_{11}^{E_{3}}\left(A^{E_{3}}+A^{E_{4}}\right) & \kappa_{12}^{E_{4}} A^{E_{4}} \\
\kappa_{12}^{E_{1}} A^{E_{1}} & 0 & \kappa_{12}^{E_{4}} A^{E_{4}} & \kappa_{22}^{E_{1}}\left(A^{E_{1}}+A^{E_{4}}\right)
\end{array}\right] \\
\mathbf{B}=\frac{1}{2}\left[\begin{array}{cccc}
\Delta y^{E_{1}} & -\Delta y^{E_{1}} & 0 & 0 \\
0 & \Delta x^{E_{2}} & -\Delta x^{E_{2}} & 0 \\
0 & 0 & -\Delta y^{E_{3}} & \Delta y^{E_{3}} \\
\Delta x^{E_{4}} & 0 & 0 & -\Delta x^{E_{4}}
\end{array}\right]
\end{gathered}
$$

The solution was implemented in fortran over a nonhomogeneous rectangular mesh having $\mathrm{nm}$ cells and $(n+$ $1)(m+1)$ nodes adopting the equation based method. Traditionally the pressure field is solved at the center of the each element, while the velocities are solved on the edges. While the TPFA solves for one velocity per edge, the MPFA solves for two for each edge, both will be calculated and an average for each edge will be found. There will therefore be $(m+1) \cdot n$ horizontal velocities, $(n+1) \cdot m$ vertical velocities and $m \cdot n$ pressures over the whole domain. The filling convention used for the mesh is rowwise: from left to right and from the bottom to the top. Figure 5 shows the filling convention for the grid. The pressure and velocity boundary conditions are imposed naturally at the extreme centers and edges of the grid respectively.

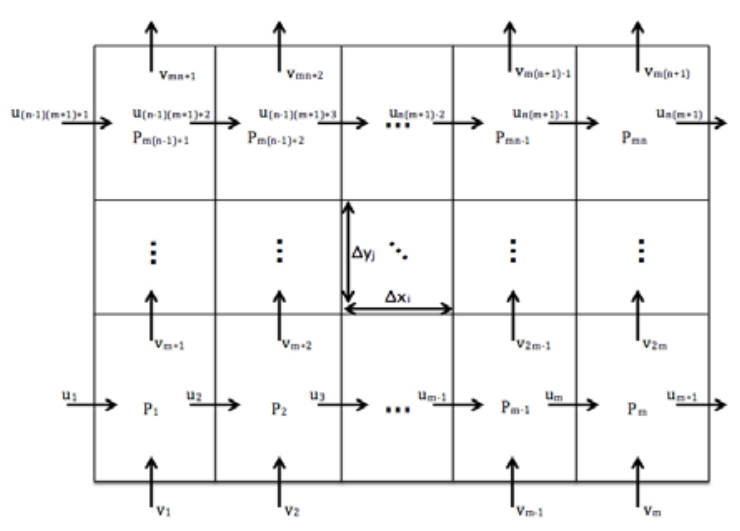

FIGURE 5. Filling convention. The velocities are calculated at the edges while pressures are calculated at the center of each cell.

\section{NUMERICAL EXAMPLES}

For the sake of illustration we apply the MPFA technique to the problem of flow in a 2D homogeneous porous medium on a rectangular domain of size $500 \mathrm{~m} \times 100 \mathrm{~m}$. The domain is subject to the following boundary conditions: the West and the East sides are assigned a pressure condition of $2 \times 10^{5} \mathrm{~Pa}$ and $1 \times 10^{5} \mathrm{~Pa}$ respectively, while the North and South boundaries are no flow. We assume four scenarios of permeability anisotropy. In the first scenario the direction of anisotropy is at $0^{\circ}$ with the positive direction of the horizontal axis (x-direction) which replicates a scenario with no anisotropy. For the other scenarios we consider an angle of $30^{\circ}, 45^{\circ}, 60^{\circ}$ and $135^{\circ}$ with the positive $\mathrm{x}$-direction. That is the largest permeability is aligned with the direction of anisotropy and the smallest is normal to it. In this work we assume an order of magnitude difference between both permeability. Figure 6 below shows a schematic diagram of the computational domain, the boundary conditions and the anisotropy direction. To implement the MPFA technique on these examples, the computational domain is discretized into a $500 \times 100$ rectangular cells (i.e., a $1 \times 1$ $m^{2}$ cells).

The results of simulation are shown in Figures 7-11 for the four cases. The results show that, while in homogeneous, isotropic system the pressure contours are expected to be parallel to the West and East boundaries (i.e., normal to the North and the South boundaries), however for these systems the contours propagate parallel to the direction of anisotropy. Furthermore the x-velocity profiles at the west boundary (i.e., at the inlet) are not uniformly distributed along the boundary as would have been expected for the homogeneous, isotropic case; a direct manifestation of the influence of anisotropy on flow field. Furthermore, the velocity profiles are shown to be 
greater at the corner where anisotropy meet. This is because the hydraulic conductivity along the anisotropy plane is the maximum and therefore draws most of the flow towards the anisotropy diection.

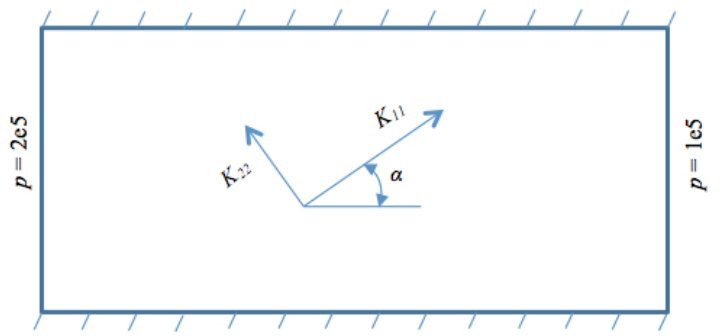

FIGURE 6. Schematic of the computational domain.

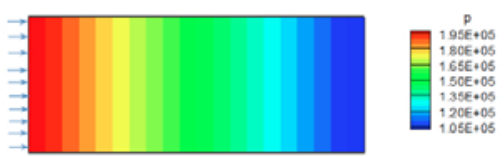

FIGURE 7. Pressure contours, $\alpha=0^{\circ}$.
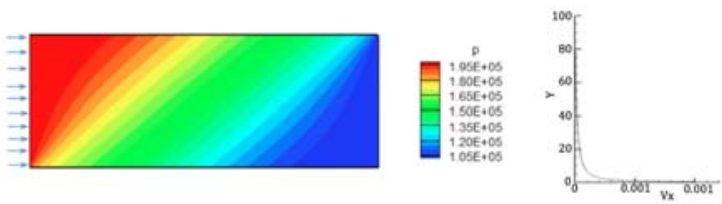

FIGURE 8. Left: Pressure contours, $\alpha=30^{\circ}$. Right: $\mathrm{x}$ velocity profile at the inlet
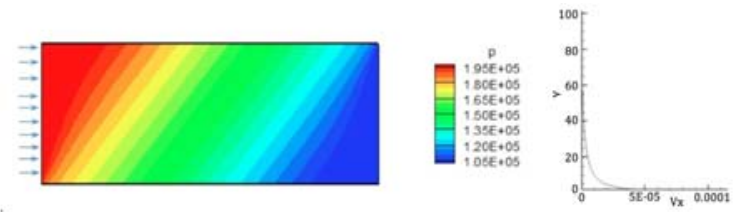

FIGURE 9. Left: Pressure contours, $\alpha=45^{\circ}$. Right: $\mathrm{x}$ velocity profile at the inlet
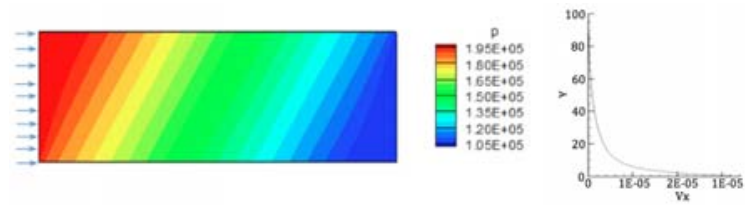

FIGURE 10. Left: Pressure contours, $\alpha=60^{\circ}$. Right: $\mathrm{x}$ velocity profile at the inlet

\section{CONCLUSIONS}

In this paper we presented the multipoint flux numerical approach to the problem of flow in anisotropic porous
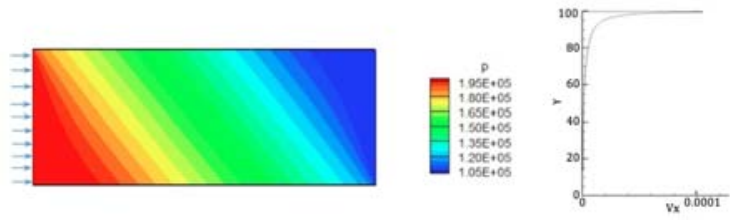

FIGURE 11. Left: Pressure contours, $\alpha=135^{\circ}$. Right: $\mathrm{x}-$ velocity profile at the inlet

media. In this work, the BDM1-based mixed finite element method is transformed with a quadrature rule to a cell-cemtered finite difference method, CCFD. Apart from the twopoint flux approximation in the usual CCFD in which the flux at face center requires two points, in this technique the flux at face center require siz points to account for anisotropy. Moreover this technique reduces naturally to the twopoint flux approximation in homogeneous media. We implemented this technique on several anisotropy scenarios with angle inclination $0^{\circ}, 30^{\circ}$, $45^{\circ}, 60^{\circ}$ and $135^{\circ}$. The pressure and velocity fields for these scenarios are shown to be significantly affected by anisotropy.

\section{REFERENCES}

1. A. Sahin, H. Menouar, A. Ali, and S.Saner, "Patterns of Variation of Permeability Anisotropy in a Carbonate Reservoir," in SPE Middle East Oil Show and Conference, Bahrain, 2003.

2. H. Al-Hadrami, and L. Teufel, "Influence of Permeability Anistropy and Reservoir Heterogeneity on Optimization of Infill Drilling in Naturally Fractured Tight-Gas Mesaverde Sandstone Reservoirs, San Juan Basin," in SPE Rocky Mountain Regional/Low Permeability Reservoir Symposium, Denver, 2000.

3. T. Blanton, "An Experimental Study of Interaction between Hydraulically Induced and Pre-Existing Fractures," in SPE Unconventional Gas Recovery Symposium, Pittsburgh, PA, 1982.

4. I. Aavatsmark, Computational Geosciences 6, 405-432 (2002).

5. M. Wheeler, and I. Yotov, IMA Volumes in Mathematics and its Applications 142, 189-208 (2006).

6. C. Ayan, N. Colley, G. Cowan, E. Ezekwe, M. Wannell, P. Goode, Santos, F. Halford, J. Joseph, A. Mongini, G. Obondoko, and J. Pop, Oilfield Review 6 (1994).

7. A. Salama, and P. Van-Geel, Journal of Porous Media 11, 403-413 (2008).

8. A. Salama, and P. Van-Geel, Journal of Porous Media 11, 421-441 (2008).

9. Z. Chen, Finite Element Methods and their Applications, Springer, Germany, 2005, 1st edn. 